\title{
Enhanced information processing at revisited fixation locations
}

Andrey R. Nikolaev ${ }^{1 *}$, Marcello Giannini ${ }^{1}$, Radha Nila Meghanathan ${ }^{1,2}$, Cees van Leeuwen ${ }^{1,2}$

${ }^{1}$ Laboratory for Perceptual Dynamics, Brain \& Cognition Research Unit, KU Leuven -

University of Leuven, Leuven, Belgium

${ }^{2}$ Center for Cognitive Science, Technical University, Kaiserslautern, Germany

* Corresponding author

Laboratory for Perceptual Dynamics,

Brain \& Cognition Research Unit,

KU Leuven - University of Leuven,

Tiensestraat 102, Box 3711,

3000 Leuven

Belgium

Andrey.Nikolaev@kuleuven.be

\section{Withdrawal state ment}

The authors have withdrawn this manuscript because of the issue in the data analysis, which was found during revision. Specifically, not all eye movement characteristics were matched between experimental conditions of interest, and these characteristics were confounded with the main EEG findings. When the issue was corrected the main effects were gone. Therefore, the authors do not wish this work to be cited. If you have any questions, please contact the corresponding author. 